\title{
WHICH SOLUTIONS OF THE THIRD PROBLEM FOR THE POISSON EQUATION ARE BOUNDED?
}

\author{
DAGMAR MEDKOVÁ
}

Received 10 September 2002

This paper deals with the problem $\Delta u=g$ on $G$ and $\partial u / \partial n+u f=L$ on $\partial G$. Here, $G \subset \mathbb{R}^{m}$, $m>2$, is a bounded domain with Lyapunov boundary, $f$ is a bounded nonnegative function on the boundary of $G, L$ is a bounded linear functional on $W^{1,2}(G)$ representable by a real measure $\mu$ on the boundary of $G$, and $g \in L_{2}(G) \cap L_{p}(G), p>m / 2$. It is shown that a weak solution of this problem is bounded in $G$ if and only if the Newtonian potential corresponding to the boundary condition $\mu$ is bounded in $G$.

Suppose that $G \subset \mathbb{R}^{m}, m>2$, is a bounded domain with Lyapunov boundary (i.e., of class $\left.C^{1+\alpha}\right)$. Denote by $n(y)$ the outer unit normal of $G$ at $y$. If $f, g, h \in C(\partial G)$ and $u \in C^{2}(\mathrm{cl} G)$ is a classical solution of

$$
\begin{gathered}
\Delta u=g \quad \text { on } G \\
\frac{\partial u}{\partial n}+u f=h \quad \text { on } \partial G
\end{gathered}
$$

then Green's formula yields

$$
\int_{G} \nabla u \cdot \nabla v d \mathscr{H}_{m}+\int_{\partial G} u f v d \mathscr{H}_{m-1}=\int_{\partial G} h v d \mathscr{H}_{m-1}-\int_{G} g v d \mathscr{H}_{m}
$$

for each $v \in \mathscr{D}$, the space of all compactly supported infinitely differentiable functions in $\mathbb{R}^{m}$. Here, $\partial G$ denotes the boundary of $G$ and $c l G$ is the closure of $G$; $\mathscr{H}_{k}$ is the $k$ dimensional Hausdorff measure normalized so that $\mathscr{H}_{k}$ is the Lebesgue measure in $\mathbb{R}^{k}$. Denote by $\mathscr{D}(G)$ the set of all functions from $\mathscr{D}$ with the support in $G$.

For an open set $V \subset \mathbb{R}^{m}$, denote by $W^{1,2}(V)$ the collection of all functions $f \in L_{2}(V)$, the distributional gradient of which belongs to $\left[L_{2}(V)\right]^{m}$.

Definition 1. Let $f \in L_{\infty}(\mathscr{H}), g \in L_{2}(G)$ and let $L$ be a bounded linear functional on $W^{1,2}(G)$ such that $L(\varphi)=0$ for each $\varphi \in \mathscr{D}(G)$. We say that $u \in W^{1,2}(G)$ is a weak solution 
in $W^{1,2}(G)$ of the third problem for the Poisson equation

$$
\begin{gathered}
\Delta u=g \quad \text { on } G \\
\frac{\partial u}{\partial n}+u f=L \quad \text { on } \partial G
\end{gathered}
$$

if

$$
\int_{G} \nabla u \cdot \nabla v d \mathscr{H}_{m}+\int_{\partial G} u f v d \mathscr{H}=L(v)-\int_{G} g v d \mathscr{H}_{m}
$$

for each $v \in W^{1,2}(G)$.

Denote by $\mathscr{C}^{\prime}(\partial G)$ the Banach space of all finite signed Borel measures with support in $\partial G$ with the total variation as a norm. We say that the bounded linear functional $L$ on $W^{1,2}(G)$ is representable by $\mu \in \mathscr{C}^{\prime}(\partial G)$ if $L(\varphi)=\int \varphi d \mu$ for each $\varphi \in \mathscr{D}$. Since $\mathscr{D}$ is dense in $W^{1,2}(G)$, the operator $L$ is uniquely determined by its representation $\mu \in \mathscr{C}^{\prime}(\partial G)$.

For $x, y \in \mathbb{R}^{m}$, denote

$$
h_{x}(y)= \begin{cases}(m-2)^{-1} A^{-1}|x-y|^{2-m} & \text { for } x \neq y \\ \infty & \text { for } x=y\end{cases}
$$

where $A$ is the area of the unit sphere in $\mathbb{R}^{m}$. For the finite real Borel measure $\nu$, denote

$$
\mathcal{u}(x)=\int_{\mathbb{R}^{m}} h_{x}(y) d \nu(y)
$$

the Newtonian potential corresponding to $v$, for each $x$ for which this integral has sense.

We denote by $\mathscr{C}_{b}^{\prime}(\partial G)$ the set of all $\mu \in \mathscr{C}^{\prime}(\partial G)$ for which $\mathscr{U} \mu$ is bounded on $\mathbb{R}^{m} \backslash \partial G$.

Remark that $\mathscr{C}_{b}^{\prime}(\partial G)$ is the set of all $\mu \in \mathscr{C}^{\prime}(\partial G)$ for which there is a polar set $M$ such that $U \mu(x)$ is meaningful and bounded on $\mathbb{R}^{m} \backslash M$, because $\mathbb{R}^{m} \backslash \partial G$ is finely dense in $\mathbb{R}^{m}$ (see [1, Chapter VII, Sections 2, 6], [7, Theorems 5.10 and 5.11]) and $u \mu=u \mu^{+}-u_{\mu}{ }^{-}$ is finite and fine-continuous outside of a polar set. Remark that $\mathscr{H}_{m-1}(M)=0$ for each polar set $M$ (see [7, Theorem 3.13]). (For the definition of polar sets, see [4, Chapter 7, Section 1]; for the definition of the fine topology, see [4, Chapter 10].)

Denote by $\mathscr{H}$ the restriction of $\mathscr{H}_{m-1}$ to $\partial G$.

Lemma 2. Let $\mu \in \mathscr{C}^{\prime}(\partial G)$. Then the following assertions are equivalent:

(1) $\mu \in \mathscr{C}_{b}^{\prime}(\partial G)$

(2) $U \mu$ is bounded in $G$,

(3) $u_{\mu} \in L_{\infty}(\mathscr{H})$.

Proof. (2) $\Rightarrow(3)$. Since $\partial G$ is a subset of the fine closure of $G$ by [1, Chapter VII, Sections 2,6] and [7, Theorems 5.10 and 5.11], $U \mu=\mho \mu^{+}-\vartheta \mu^{-}$is finite and fine-continuous outside of a polar set $M$, and $\mathscr{H}_{m-1}(M)=0$ by [4, Theorem 7.33] and [7, Theorem 3.13], then we obtain that $u_{\mu} \in L_{\infty}(\mathscr{H})$. 
(3) $\Rightarrow(1)$. Let $\mu=\mu^{+}-\mu^{-}$be the Jordan decomposition of $\mu$. For $z \in G$, denote by $\mu_{z}$ the harmonic measure corresponding to $G$ and $z$. If $y \in \partial G$ and $z \in G$, then

$$
\int_{\partial G} h_{y}(x) d \mu_{z}(x)=h_{y}(z)
$$

by [7, pages 264, 299]. Using Fubini's theorem, we get

$$
\int u \mu^{+} d \mu_{z}=\int_{\partial G} \int_{\partial G} h_{y}(x) d \mu_{z}(x) d \mu^{+}(y)=\int_{\partial G} h_{y}(z) d \mu^{+}(y)=u \mu^{+}(z) .
$$

Similarly, $\int u \mu^{-} d \mu_{z}=\vartheta \mu^{-}(z)$. Since $u \mu \in L_{\infty}(\mathscr{H}), \mu_{z}$ is a nonnegative measure with the total variation 1 (see [4, Lemma 8.12]) which is absolutely continuous with respect to $\mathscr{H}$ by $\left[2\right.$, Theorem 1], then we obtain that $|\Theta \mu(z)| \leq\left\|u_{\mu}\right\|_{L_{\infty}(\mathscr{H})}$.

If $z \in \mathbb{R}^{m} \backslash \mathrm{cl} G$, choose a bounded domain $V$ with smooth boundary such that $\mathrm{cl} G \cup$

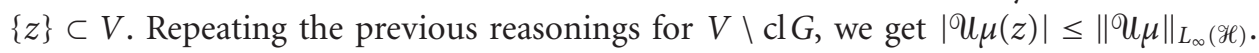

Lemma 3. Let $f \in L_{\infty}(\mathscr{H})$ and $g \in L_{2}(G) \cap L_{p}\left(\mathbb{R}^{m}\right)$, where $p>m / 2, g=0$ on $\mathbb{R}^{m} \backslash G$. Then $\mathcal{U}\left(g^{\mathcal{H}_{m}}\right) \in \mathscr{C}\left(\mathbb{R}^{m}\right) \cap W^{1,2}(G)$. Moreover, there is a bounded linear functional $L$ on $W^{1,2}(G)$ representable by $\mu \in \mathscr{C}_{b}^{\prime}(\partial G)$ such that $\mathscr{U}\left(\mathrm{g}_{\mathcal{H}}\right)$ is a weak solution in $W^{1,2}(G)$ of the third problem for the Poisson equation

$$
\Delta u=-g \quad \text { on } G, \quad \frac{\partial u}{\partial n}+u f=L \quad \text { on } \partial G .
$$

Proof. Suppose first that $g$ is nonnegative. Since $U\left(g \mathscr{H}_{m}\right) \in \mathscr{C}\left(\mathbb{R}^{m}\right)$ by [3, Theorem A.6], the energy $\int g^{\mathscr{U}}\left(g_{\mathcal{H}}\right) d \mathscr{H}_{m}<\infty$. According to [7, Theorem 1.20], we have

$$
\int\left|\nabla \mathcal{U}\left(g \mathscr{H}_{m}\right)\right|^{2} d \mathscr{H}_{m}=\int g \mathcal{U}\left(g \mathscr{H}_{m}\right) d \mathscr{H}_{m}<\infty,
$$

and therefore $\mathcal{U}\left(g_{\mathcal{H}_{m}}\right) \in W^{1,2}(G)$ (see [7, Lemma 1.6] and [16, Theorem 2.1.4]).

Since $\mathcal{U}\left(g_{\mathscr{H}_{m}}\right) \in \mathscr{C}\left(\mathbb{R}^{m}\right) \cap W^{1,2}(G), f \in L_{\infty}(\mathscr{H})$ and the trace operator is a bounded operator from $W^{1,2}(G)$ to $L_{2}(\mathscr{H})$ by $[8$, Theorem 3.38], then the operator

$$
L(\varphi)=\int_{G} \nabla \varphi \cdot \nabla \mathcal{U}\left(g_{\mathcal{H}_{m}}\right) d \mathscr{H}_{m}+\int_{\partial G} \mathcal{U}\left(g^{\mathscr{H}_{m}}\right) f \varphi d \mathscr{H}_{m-1}-\int_{G} g \varphi d \mathscr{H}_{m}
$$

is a bounded linear functional on $W^{1,2}(G)$.

According to [7, Theorem 4.2], there is a nonnegative $v \in \mathscr{C}^{\prime}(\partial G)$ such that $\mathcal{U} v=$ $\mathcal{U}\left(g_{\mathcal{H}}\right)$ on $\mathbb{R}^{m} \backslash \mathrm{cl} G$. Choose a bounded domain $V$ with smooth boundary such that $\mathrm{cl} G \subset V$. Since $\mathscr{U} \nu$ is bounded in $V \backslash \mathrm{cl} G \subset \mathbb{R}^{m} \backslash \mathrm{cl} G$, Lemma 2 yields that $\nu \in \mathscr{C}_{b}^{\prime}(\partial(V \backslash$ $\mathrm{cl} G)$ ). Therefore, $v \in \mathscr{C}_{b}^{\prime}(\partial G)$. According to [13, Lemma 4], there is $\tilde{\nu} \in \mathscr{C}_{b}^{\prime}(\partial G)$ such that

$$
\int_{\mathbb{R}^{m} \backslash \mathrm{cl} G} \nabla \varphi \cdot \nabla \mathcal{U}\left(g \mathscr{H}_{m}\right) d \mathscr{H}_{m}=\int_{\mathbb{R}^{m} \backslash \mathrm{cl} G} \nabla \varphi \cdot \nabla \mathcal{U} \nu d \mathcal{H}_{m}=\int_{\partial G} \varphi d \tilde{\nu}
$$

for each $\varphi \in \mathscr{D}$. Let $\mu=\tilde{v}-f \cup\left(g^{\mathcal{H}_{m}}\right) \mathscr{H}$. Since $\mathcal{U}\left(f \mathcal{U}\left(g_{\mathcal{H}_{m}}\right) \mathscr{H}\right) \in \mathscr{C}\left(\mathbb{R}^{m}\right)$ by $[6$, Corollary

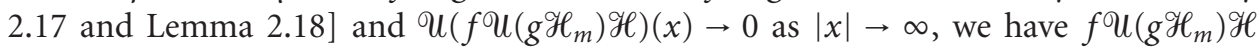
$\in \mathscr{C}_{b}^{\prime}(\partial G)$. Therefore, $\mu \in \mathscr{C}_{b}^{\prime}(\partial G)$. 
If $\varphi \in \mathscr{D}$, then $\varphi=\mathcal{U}\left((-\Delta \varphi) \mathscr{H}_{m}\right)$ by [3, Theorem A.2]. According to [7, Theorem $1.20]$,

$$
\begin{aligned}
\int_{\mathbb{R}^{m}} \nabla \varphi \cdot \nabla \mathcal{U}\left(g \mathscr{H}_{m}\right) d \mathscr{H}_{m} & =\int_{\mathbb{R}^{m}} \nabla \mathcal{U}\left((-\Delta \varphi) \mathscr{H}_{m}\right) \cdot \nabla \mathcal{U}\left(g \mathscr{H}_{m}\right) d \mathscr{H}_{m} \\
& =\int_{\mathbb{R}^{m}} g \mathcal{U}\left((-\Delta \varphi) \mathscr{H}_{m}\right) d \mathscr{H}_{m} \\
& =\int_{\mathbb{R}^{m}} g \varphi d \mathscr{H}_{m} .
\end{aligned}
$$

Since $\mathscr{H}_{m}(\partial G)=0$

$$
\begin{aligned}
& \int_{G} \nabla \varphi \cdot \nabla \mathcal{U}\left(g^{\mathcal{H}_{m}}\right) d \mathscr{H}_{m}+\int_{\partial G} \boldsymbol{u}\left(g^{\mathcal{H}_{m}}\right) f \varphi d \mathscr{H}_{m-1} \\
& =\int_{G} g \varphi d \mathscr{H}_{m}+\int_{\partial G} u\left(g \mathscr{H}_{m}\right) f \varphi d \mathscr{H}_{m-1} \\
& -\int_{\mathbb{R}^{m} \backslash \mathrm{cl} G} \nabla \varphi \cdot \nabla \vartheta\left(g \mathscr{H}_{m}\right) d \mathscr{H}_{m} \\
& =\int_{G} g \varphi d \mathscr{H}_{m}+\int_{\partial G} \varphi d \mu \text {. }
\end{aligned}
$$

Lemma 4. Let $f \in L_{\infty}(\mathscr{H})$ and $g \in L_{2}(G) \cap L_{p}\left(\mathbb{R}^{m}\right)$, where $p>m / 2, g=0$ on $\mathbb{R}^{m} \backslash G$. Let $L$ be a bounded linear functional on $W^{1,2}(G)$ representable by $\mu \in \mathscr{G}^{\prime}(\partial G)$. If $u \in L_{\infty}(G) \cap$ $W^{1,2}(G)$ is a weak solution in $W^{1,2}(G)$ of problem (3), then $\mu \in \mathscr{C}_{b}^{\prime}(\partial G)$.

Proof. Let $w=u-\mathcal{U}\left(g_{\mathcal{H}}\right)$. According to Lemma 3, there is a bounded linear functional $\tilde{L}$ on $W^{1,2}(G)$ representable by $v \in \mathscr{C}_{b}^{\prime}(\partial G)$ such that $w$ is a weak solution in $W^{1,2}(G)$ of the problem

$$
\begin{aligned}
\Delta w & =0 \quad \text { on } G \\
\frac{\partial w}{\partial n}+w f & =L-\tilde{L} \quad \text { on } \partial G .
\end{aligned}
$$

Fix $x \in G$. Choose a sequence $G_{j}$ of open sets with $C^{\infty}$ boundary such that $\mathrm{cl}_{j} \subset G_{j+1} \subset$ $G, x \in G_{1}$, and $\cup G_{j}=G$. Fix $r>0$ such that $\Omega_{2 r}(x) \subset G_{1}$. Choose an infinitely differentiable function $\psi$ such that $\psi=0$ on $\Omega_{r}(x)$ and $\psi=1$ on $\mathbb{R}^{m} \backslash \Omega_{2 r}(x)$. According to Green's identity,

$$
\begin{aligned}
& w(x)=\lim _{j \rightarrow \infty} {\left[\int_{\partial G_{j}} h_{x}(y) \frac{\partial w(y)}{\partial n} d \mathscr{H}_{m-1}(y)-\int_{\partial G_{j}} w(y) n(y) \cdot \nabla h_{x}(y) d \mathscr{H}_{m-1}(y)\right] } \\
&=\lim _{j \rightarrow \infty}\left[\int_{G_{j}} \nabla w(y) \cdot \nabla\left(h_{x}(y) \psi(y)\right) d \mathscr{H}_{m}(y)\right. \\
&\left.-\int_{G_{j}} \nabla(w(y) \psi(y)) \cdot \nabla h_{x}(y) d \mathscr{H}_{m}(y)\right]
\end{aligned}
$$




$$
\begin{aligned}
& =\int_{G} \nabla w(y) \cdot \nabla\left(h_{x}(y) \psi(y)\right) d \mathscr{H}_{m}(y)-\int_{G} \nabla(w(y) \psi(y)) \cdot \nabla h_{x}(y) d \mathscr{H}_{m}(y) \\
& =\mathcal{U}(\mu-v-f w \mathscr{H})(x)-\int_{G} \nabla(w(y) \psi(y)) \cdot \nabla h_{x}(y) d \mathscr{H}_{m}(y) .
\end{aligned}
$$

According to [16, Theorem 2.3.2], there is a sequence of infinitely differentiable functions $w_{n}$ such that $w_{n} \rightarrow w \psi$ in $W^{1,2}(G)$. According to [6, Section 2],

$$
\begin{aligned}
w(x) & =\mathcal{U}(\mu-v-f w \mathscr{H})(x)-\lim _{n \rightarrow \infty} \int_{G} \nabla w_{n}(y) \cdot \nabla h_{x}(y) d \mathscr{H}_{m}(y) \\
& =U(\mu-v-f w \mathscr{H})(x)-\lim _{n \rightarrow \infty} \int_{\partial G} w_{n}(y) n(y) \cdot \nabla h_{x}(y) d \mathscr{H}_{m-1}(y) .
\end{aligned}
$$

Since the trace operator is a bounded operator from $W^{1,2}(G)$ to $L_{2}(\mathscr{H})$ by $[8$, Theorem 3.38], we obtain

$$
w(x)=\mathcal{U}(\mu-v-f w \mathscr{H})(x)-\int_{\partial G} w(y) n(y) \cdot \nabla h_{x}(y) d \mathscr{H}_{m-1}(y) .
$$

Since $w \in L_{\infty}(G)$ by Lemma 3, the trace of $w$ is an element of $L_{\infty}(\mathscr{H})$. Since

$$
\begin{aligned}
& \left|\int_{\partial G} w(y) n(y) \cdot \nabla h_{x}(y) d \mathscr{H}_{m-1}(y)\right| \\
& \quad \leq\|w\|_{L_{\infty}(\mathscr{H})} \int_{\partial G}\left|n(y) \cdot \nabla h_{x}(y)\right| d \mathscr{H}_{m-1}(y) \\
& \quad \leq\|w\|_{L_{\infty}(\mathscr{H})}\left[\sup _{z \in \partial G} \int_{\partial G}\left|n(y) \cdot \nabla h_{z}(y)\right| d \mathscr{H}_{m-1}(y)+\frac{1}{2}\right]<\infty
\end{aligned}
$$

by [6, Lemma 2.15 and Theorem 2.16] and the fact that $\partial G$ is of class $C^{1+\alpha}$, the function

$$
x \longmapsto \int_{\partial G} w(y) n(y) \cdot \nabla h_{x}(y) d \mathscr{H}_{m-1}(y)
$$

is bounded in $G$. Since $\mathcal{U} \nu$ is bounded in $G$ and $\mathcal{U}(f w \mathscr{H})$ is bounded in $G$ by [6, Corollary 2.17 and Lemma 2.18], the function $\mathcal{U}_{\mu}$ is bounded in $G$ by (18). Thus, $\mu \in \mathscr{C}_{b}^{\prime}(\partial G)$ by Lemma 2.

Notation 5. Let $X$ be a complex Banach space and $T$ a bounded linear operator on $X$. We denote by $\operatorname{Ker} T$ the kernel of $T$, by $\sigma(T)$ the spectrum of $T$, by $r(T)$ the spectral radius of $T$, by $X^{\prime}$ the dual space of $X$, and by $T^{\prime}$ the adjoint operator of $T$. Denote by $I$ the identity operator.

Theorem 6. Let $X$ be a complex Banach space and $K$ a compact linear operator on $X$. Let $Y$ be a subspace of $X^{\prime}$ and $T$ a closed linear operator from $Y$ to $X$ such that $y(T x)=x(T y)$ for each $x, y \in Y$. Suppose that $K^{\prime}(Y) \subset Y$ and $K T y=T K^{\prime} y$ for each $y \in Y$. Let $\alpha \in C \backslash\{0\}$, $\operatorname{Ker}\left(K^{\prime}-\alpha I\right)^{2}=\operatorname{Ker}\left(K^{\prime}-\alpha I\right) \subset Y$, and $\left\{\beta \in \sigma\left(K^{\prime}\right) ;(\beta-\alpha) \cdot \alpha \leq 0\right\} \subset\{\alpha\}$. If $x, y \in X$, $\left(K^{\prime}-\alpha I\right) x=y$, then $x \in Y$ if and only if $y \in Y$. 
Proof. If $x \in Y$, then $y \in Y$. Suppose that $y \in Y$. Since $K$ is a compact operator, the operator $K^{\prime}$ is a compact operator by [14, Chapter IV, Theorem 4.1]. Suppose first that $\alpha \in \sigma\left(K^{\prime}\right)$. Since $K^{\prime}$ is compact, then $\alpha$ is a pol of the resolvent by [5, Satz 50.4]. Since

$$
\operatorname{Ker}\left(K^{\prime}-\alpha I\right)^{2}=\operatorname{Ker}\left(K^{\prime}-\alpha I\right)
$$

the ascent of $\left(K^{\prime}-\alpha I\right)$ is equal to 1 . Since $\alpha$ is a pol of the resolvent and the ascent of $\left(K^{\prime}-\alpha I\right)$ is equal to 1 , [5, Satz 50.2] yields that the space $X^{\prime}$ is the direct sum of $\operatorname{Ker}\left(K^{\prime}-\right.$ $\alpha I)$ and $\left(K^{\prime}-\alpha I\right)\left(X^{\prime}\right)$ and the descent of $\left(K^{\prime}-\alpha I\right)$ is equal to 1 . Since the descent of $\left(K^{\prime}-\alpha I\right)$ is equal to 1 , we have

$$
\left(K^{\prime}-\alpha I\right)^{2}\left(X^{\prime}\right)=\left(K^{\prime}-\alpha I\right)\left(X^{\prime}\right) .
$$

Since the space $X^{\prime}$ is the direct sum of $\operatorname{Ker}\left(K^{\prime}-\alpha I\right)$ and $\left(K^{\prime}-\alpha I\right)\left(X^{\prime}\right)=\left(K^{\prime}-\alpha I\right)^{2}\left(X^{\prime}\right)$, the operator $\left(K^{\prime}-\alpha I\right)$ is invertible on $\left(K^{\prime}-\alpha I\right)\left(X^{\prime}\right)$. If $\alpha \notin \sigma\left(K^{\prime}\right)$, then the space $X^{\prime}$ is the direct sum of $\operatorname{Ker}\left(K^{\prime}-\alpha I\right)$ and $\left(K^{\prime}-\alpha I\right)\left(X^{\prime}\right)$, and the operator $\left(K^{\prime}-\alpha I\right)$ is invertible on $\left(K^{\prime}-\alpha I\right)\left(X^{\prime}\right)$. Therefore, there are $x_{1} \in \operatorname{Ker}\left(K^{\prime}-\alpha I\right) \subset Y$ and $x_{2} \in\left(K^{\prime}-\alpha I\right)\left(X^{\prime}\right)$ such that $x_{1}+x_{2}=x$. We have $\left(K^{\prime}-\alpha I\right) x_{2}=y$.

Denote by $Z$ the closure of $Y$. Since $K^{\prime}(Y) \subset Y$, we obtain $K^{\prime}(Z) \subset Z$. Denote by $K_{Z}^{\prime}$ the restriction of $K^{\prime}$ to $Z$. Then $K_{Z}^{\prime}$ is a compact operator in $Z$. Since $\operatorname{Ker}\left(K^{\prime}-\alpha I\right)^{2} \subset Y$, we have

$$
\operatorname{Ker}\left(K_{Z}^{\prime}-\alpha I\right)^{2}=\operatorname{Ker}\left(K^{\prime}-\alpha I\right)^{2}=\operatorname{Ker}\left(K^{\prime}-\alpha I\right)=\operatorname{Ker}\left(K_{Z}^{\prime}-\alpha I\right)
$$

If $\alpha \notin \sigma\left(K_{Z}^{\prime}\right)$, then the space $Z$ is the direct sum of $\operatorname{Ker}\left(K_{Z}^{\prime}-\alpha I\right)$ and $\left(K_{Z}^{\prime}-\alpha I\right)(Z)$, and the operator $\left(K_{Z}^{\prime}-\alpha I\right)$ is invertible on $Z$. Suppose that $\alpha \in \sigma\left(K_{Z}^{\prime}\right)$. Since $K_{Z}^{\prime}$ is compact, then $\alpha$ is a pol of the resolvent by [5, Satz 50.4]. Since

$$
\operatorname{Ker}\left(K_{Z}^{\prime}-\alpha I\right)^{2}=\operatorname{Ker}\left(K_{Z}^{\prime}-\alpha I\right)
$$

the ascent of $\left(K_{Z}^{\prime}-\alpha I\right)$ is equal to 1 . Since $\alpha$ is a pol of the resolvent and the ascent of $\left(K_{Z}^{\prime}-\alpha I\right)$ is equal to 1 , [5, Satz 50.2] yields that the space $Z$ is the direct sum of $\operatorname{Ker}\left(K_{Z}^{\prime}-\right.$ $\alpha I)$ and $\left(K_{Z}^{\prime}-\alpha I\right)(Z)$ and the descent of $\left(K_{Z}^{\prime}-\alpha I\right)$ is equal to 1 . Since the descent of $\left(K_{Z}^{\prime}-\alpha I\right)$ is equal to 1 , we have

$$
\left(K_{Z}^{\prime}-\alpha I\right)^{2}(Z)=\left(K^{\prime}-\alpha I\right)(Z)
$$

Since the space $Z$ is the direct sum of $\operatorname{Ker}\left(K_{Z}^{\prime}-\alpha I\right)$ and $\left(K_{Z}^{\prime}-\alpha I\right)(Z)=\left(K_{Z}^{\prime}-\alpha I\right)^{2}(Z)$, the operator $\left(K_{Z}^{\prime}-\alpha I\right)$ is invertible on $\left(K_{Z}^{\prime}-\alpha I\right)(Z)$. Since $y \in Y \subset Z$, there are $y_{1} \in$ $\operatorname{Ker}\left(K_{Z}^{\prime}-\alpha I\right)$ and $y_{2} \in\left(K_{Z}^{\prime}-\alpha I\right)(Z)$ such that $y=y_{1}+y_{2}$. Since $X^{\prime}$ is the direct sum of $\operatorname{Ker}\left(K^{\prime}-\alpha I\right)=\operatorname{Ker}\left(K_{Z}^{\prime}-\alpha I\right)$ and $\left(K^{\prime}-\alpha I\right)\left(X^{\prime}\right) \supset\left(K_{Z}^{\prime}-\alpha I\right)(Z)$ and $y \in\left(K^{\prime}-\alpha I\right)\left(X^{\prime}\right)$, we obtain that $y_{1}=0$ and $y_{2}=y$. Thus, $y \in\left(K_{Z}^{\prime}-\alpha I\right)(Z)$. Since $\left(K_{Z}^{\prime}-\alpha I\right)$ is invertible on $\left(K_{Z}^{\prime}-\alpha I\right)(Z)$, there is $z \in\left(K_{Z}^{\prime}-\alpha I\right)(Z)$ such that $\left(K_{Z}^{\prime}-\alpha I\right)(z)=y$. Since $\left(K^{\prime}-\alpha I\right)$ is invertible on $\left(K^{\prime}-\alpha I\right)\left(X^{\prime}\right)$, we deduce that $x_{2}=z \in\left(K_{Z}^{\prime}-\alpha I\right)(Z) \subset Z$. 
Now, let $w \in \operatorname{Ker}\left(K^{\prime}-\alpha I\right)$. Fix a sequence $\left\{z_{k}\right\} \subset Y$ such that $z_{k} \rightarrow z=x_{2}$. Then

$$
\begin{aligned}
w(T y) & =y(T w)=\left[\left(K^{\prime}-\alpha I\right) x_{2}\right](T w)=\lim _{k \rightarrow \infty}\left[\left(K^{\prime}-\alpha I\right) z_{k}\right](T w) \\
& =\lim _{k \rightarrow \infty} z_{k}((K-\alpha I) T w)=\lim _{k \rightarrow \infty} z_{k}\left(T\left(K^{\prime}-\alpha I\right) w\right)=\lim _{k \rightarrow \infty} z_{k}(0)=0 .
\end{aligned}
$$

Since $w(T y)=0$ for each $w \in \operatorname{Ker}\left(K^{\prime}-\alpha I\right)$, [15, Chapter 10, Theorem 3] yields $T y \in$ $(K-\alpha I)(X)$.

Denote by $\tilde{K}^{\prime}$ the restriction of $K^{\prime}$ to $\left(K^{\prime}-\alpha I\right)(X)$. If we denote by $P$ the spectral projection corresponding to the spectral set $\{\alpha\}$ and the operator $K^{\prime}$, then $P\left(X^{\prime}\right)=\left(K^{\prime}-\right.$ $\alpha I)\left(X^{\prime}\right)$ by [5, Satz 50.2] and $\sigma\left(\tilde{K}^{\prime}\right)=\sigma\left(K^{\prime}\right) \backslash\{\alpha\}$ by [14, Chapter VI, Theorem 4.1]. Therefore,

$$
\sigma\left(\tilde{K}^{\prime}\right)=\sigma\left(K^{\prime}\right) \backslash\{\alpha\} \subset\{\beta ;(\beta-\alpha) \cdot \alpha>0\} \subset \underset{t>0}{\cup}\{\beta ;|\beta-\alpha-t \alpha|<|t \alpha|\} .
$$

Since $\left\{\beta ;\left|\beta-\alpha-t_{1} \alpha\right|<\left|t_{1} \alpha\right|\right\} \subset\left\{\beta ;\left|\beta-\alpha-t_{2} \alpha\right|<\left|t_{2} \alpha\right|\right\}$ for $0<t_{1}<t_{2}$ and $\sigma\left(\tilde{K}^{\prime}\right)$ is a compact set (see [14, Chapter VI, Theorem 1.3, and Lemma 1.5], there is $t>0$ such that $\sigma\left(\tilde{K}^{\prime}\right) \subset\{\beta ;|\beta-\alpha-t \alpha|<|t \alpha|\}$. Therefore, $r\left(\tilde{K}^{\prime}-\alpha I-t \alpha I\right)<|t \alpha|$. Since we have $r\left(t^{-1} \alpha^{-1}\left(\tilde{K}^{\prime}-\alpha I-t \alpha I\right)\right)<1$, the series

$$
V=\sum_{k=0}^{\infty}(-1)^{k}\left[t^{-1} \alpha^{-1}\left(\tilde{K}^{\prime}-\alpha I-t \alpha I\right)\right]^{k}
$$

converges. Easy calculation yields that $V$ is the inverse operator of the operator $I+$ $t^{-1} \alpha^{-1}\left(\tilde{K}^{\prime}-\alpha I-t \alpha I\right)=t^{-1} \alpha^{-1}\left(\tilde{K}^{\prime}-\alpha I\right)$. Since $t^{-1} \alpha^{-1} y=t^{-1} \alpha^{-1}\left(\tilde{K}^{\prime}-\alpha I\right) x_{2}$, we have $x_{2}=t^{-1} \alpha^{-1} V y$. Denote $z_{k}=t^{-1} \alpha^{-1}\left[-t^{-1} \alpha^{-1}\left(\tilde{K}^{\prime}-\alpha I-t \alpha I\right)\right]^{k} y$. Then

$$
x_{2}=\sum_{k=0}^{\infty} z_{k} \text {. }
$$

Since $K^{\prime}(Y) \subset Y, z_{k} \in Y$ for each $k$. Since $K T=T K^{\prime}$ on $Y$, we have $T z_{k}=t^{-1} \alpha^{-1}\left[-t^{-1} \alpha^{-1}(K\right.$ $-\alpha I-t \alpha I)]^{k} T y$.

Since $(K-\alpha I),(K-\alpha I)^{2},\left(K^{\prime}-\alpha I\right)$, and $\left(K^{\prime}-\alpha I\right)^{2}$ are Fredholm operators with index 0 (see [14, Chapter V, Theorem 3.1]), [14, Chapter VII, Theorem 3.2] yields

$$
\operatorname{dim} \operatorname{Ker}(K-\alpha I)^{2}=\operatorname{dim} \operatorname{Ker}\left(K^{\prime}-\alpha I\right)^{2}=\operatorname{dim} \operatorname{Ker}\left(K^{\prime}-\alpha I\right)=\operatorname{dim} \operatorname{Ker}(K-\alpha I),
$$

and thus $\operatorname{Ker}(K-\alpha I)^{2}=\operatorname{Ker}(K-\alpha I)$. If $\alpha \notin \sigma(K)$, then the space $X$ is the direct sum of $\operatorname{Ker}(K-\alpha I)$ and $(K-\alpha I)(X)$, and the operator $(K-\alpha I)$ is invertible on $X$. Suppose that $\alpha \in \sigma(K)$. Since $K$ is compact, then $\alpha$ is a pol of the resolvent by [5, Satz 50.4]. Since

$$
\operatorname{Ker}(K-\alpha I)^{2}=\operatorname{Ker}(K-\alpha I),
$$

the ascent of $(K-\alpha I)$ is equal to 1 . Since $\alpha$ is a pol of the resolvent and the ascent of $(K-\alpha I)$ is equal to 1 , [5, Satz 50.2] yields that the space $X$ is the direct sum of $\operatorname{Ker}(K-\alpha I)$ and $(K-\alpha I)(X)$ and the descent of $(K-\alpha I)$ is equal to 1 . Since the descent of $(K-\alpha I)$ is equal to 1 , we have $(K-\alpha I)^{2}(X)=(K-\alpha I)(X)$. Since the space $X$ is the direct sum 
of $\operatorname{Ker}(K-\alpha I)$ and $(K-\alpha I)(X)=(K-\alpha I)^{2}(X)$, the operator $(K-\alpha I)$ is invertible on $(K-\alpha I)(X)$. Denote by $\hat{K}$ the restriction of $K$ to $(K-\alpha I)(X)$. If we denote by $Q$ the spectral projection corresponding to the spectral set $\{\alpha\}$ and the operator $K$, then $Q(X)=$ $(K-\alpha I)(X)$ by [5, Satz 50.2] and $\sigma(\hat{K})=\sigma(K) \backslash\{\alpha\}$ by [14, Chapter VI, Theorem 4.1]. Since $\sigma(K)=\sigma\left(K^{\prime}\right)$ by [14, Chapter VI, Theorem 4.6], we obtain $\sigma(\hat{K}) \subset\{\beta ; \mid \beta-\alpha-$ $t \alpha|<| t \alpha \mid\}$. Therefore, $r(\widehat{K}-\alpha I-t \alpha I)<|t \alpha|$. Since $T y \in(K-\alpha X)$ and $r\left(t^{-1} \alpha^{-1}(\hat{K}-\right.$ $\alpha I-t \alpha I))<1$, the series

$$
\sum_{k=0}^{\infty} T z_{k}=\sum_{k=0}^{\infty} t^{-1} \alpha^{-1}\left[-t^{-1} \alpha^{-1}(\hat{K}-\alpha I-t \alpha I)\right]^{k} T y
$$

converges. Since $T$ is closed, $x_{2}=\sum z_{k}$, and $\sum T z_{k}$ converges, then the vector $x_{2}$ lies in $Y$, the domain of $T$.

Theorem 7. Let $f \in L_{\infty}(\mathscr{H}), f \geq 0$, and $g \in L_{2}(G) \cap L_{p}\left(\mathbb{R}^{m}\right)$, where $p>m / 2, g=0$ on $\mathbb{R}^{m} \backslash G$. Let $L$ be a bounded linear functional on $W^{1,2}(G)$ representable by $\mu \in \mathscr{C}^{\prime}(\partial G)$. If $u$ is a weak solution in $W^{1,2}(G)$ of problem (3), then $u \in L_{\infty}(G)$ if and only if $\mu \in \mathscr{C}_{b}^{\prime}(\partial G)$.

Proof. If $u \in L_{\infty}(G)$, then $\mu \in \mathscr{C}_{b}^{\prime}(\partial G)$ by Lemma 4 .

Suppose now that $\mu \in \mathscr{C}_{b}^{\prime}(\partial G)$. Let $w=u-\mathscr{U}\left(g_{\mathcal{H}}\right)$. According to Lemma 3 , there is a bounded linear functional $\tilde{L}$ on $W^{1,2}(G)$ representable by $\tilde{\mu} \in \mathscr{C}_{b}^{\prime}(\partial G)$ such that $w$ is a weak solution in $W^{1,2}(G)$ of the problem

$$
\begin{gathered}
\Delta w=0 \quad \text { on } G \\
\frac{\partial w}{\partial n}+w f=\tilde{L} \quad \text { on } \partial G .
\end{gathered}
$$

Define for $\varphi \in L_{\infty}(\mathscr{H})$ and $x \in \partial G$,

$$
T \varphi(x)=\frac{1}{2} \varphi(x)+\int_{\partial G} \varphi(y) \frac{\partial}{n(y)} h_{x}(y) d \mathscr{H}(y)+\mathcal{U}(f \varphi \mathscr{H}) .
$$

Since $\mathcal{U}(f \mathscr{H}) \in \mathscr{C}\left(\mathbb{R}^{m}\right)$ by [6, Corollary 2.17 and Lemma 2.18$]$, the operator $T$ is a bounded linear operator on $L_{\infty}(\mathscr{H})$ by [11, Proposition 8] and [6, Lemma 2.15]. The operator $T-(1 / 2) I$ is compact by [12, Theorem 20] and [6, Theorem 4.1 and Corollary 1.11]. According to [10, Theorem 1], there is $v \in \mathscr{G}^{\prime}(\partial G) \subset\left(L_{\infty}(\mathscr{H})\right)^{\prime}$ such that $T^{\prime} v=\tilde{\mu}$ and

$$
\int_{G} \nabla \mathcal{U} \cdot \nabla v d \mathscr{H}_{m}+\int_{\partial G} u v f v d \mathscr{H}=\int v d \tilde{\mu}
$$

for each $v \in \mathscr{D}$.

Remark that $\mathscr{C}^{\prime}(\partial G)$ is a closed subspace of $\left(L_{\infty}(\mathscr{H})\right)^{\prime}$. According to [11, Proposition 8], we have $T^{\prime}\left(\mathscr{C}^{\prime}(\partial G)\right) \subset \mathscr{C}^{\prime}(\partial G)$. Denote by $\tau$ the restriction of $T^{\prime}$ to $\mathscr{C}^{\prime}(\partial G)$. According to [10, Lemma 11] and [14, Chapter VI, Theorem 1.2], we have $\sigma(\tau) \subset\{\beta ; \beta \geq 0\}$. Since $\sigma\left(\tau^{\prime}\right)=\sigma(\tau)$ (see [15, Chapter VIII, Section 6, Theorem 2]), each $\beta \in \sigma(T)$ is an eigenvalue (see [14, Chapter VI, Theorem 1.2]), and T is the restriction of $\tau^{\prime}$ to $L_{\infty}(\mathscr{H})$, we obtain that $\sigma\left(T^{\prime}\right)=\sigma(T) \subset\{\beta ; \beta \geq 0\}$ by [15, Chapter VIII, Section 6, Theorem 2]. 
According to [9, Theorem 1.11], we have $\operatorname{Ker} T^{\prime} \subset \mathscr{C}_{b}^{\prime}(\partial G)$. According to [9, Lemma 1.10] and [10, Lemmas 12 and 13], $\operatorname{Ker} T^{\prime}=\operatorname{Ker}\left(T^{\prime}\right)^{2}$. Denote, for $\rho \in \mathscr{C}_{b}^{\prime}(\partial G)$, by $V \rho$ the restriction of $\boldsymbol{U}_{\rho}$ to $\partial G$. Then $V$ is a closed operator from $\mathscr{C}_{b}^{\prime}(\partial G)$ to $L_{\infty}(\mathscr{H})$ by $[13$, Lemma 5]. If $\rho \in \mathscr{C}_{b}^{\prime}(\partial G)$, then $V T^{\prime} \rho=T V \rho$ by [13, Lemma 4]. If $\rho_{1}, \rho_{2} \in \mathscr{C}_{b}^{\prime}(\partial G)$, then $\rho_{1}$ and $\rho_{2}$ have finite energy by [13, Proposition 23], [7, Theorem 1.20], and

$$
\int u \rho_{1} d \rho_{2}=\int_{\mathbb{R}^{m}} \nabla u \rho_{1} \cdot \nabla u \rho_{2} d \mathscr{H}_{m}=\int u \rho_{2} d \rho_{1} \text {. }
$$

Since $T^{\prime} v=\tilde{\mu} \in \mathscr{C}_{b}^{\prime}(\partial G)$, Theorem 6 yields that $v \in \mathscr{C}_{b}^{\prime}(\partial G)$. Since $v$ has finite energy

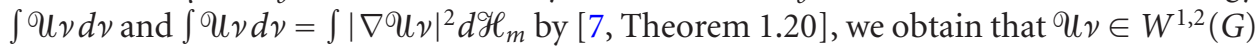
(see [7, Lemma 1.6] and [16, Theorem 2.14]). Since $\mathscr{D}$ is dense in $W^{1,2}(G)$ by [16, Theorem 2.3.2], relation (35) yields that the function $U v$ is a weak solution in $W^{1,2}(G)$ of (33). Since $v=U v-w$ is a weak solution in $W^{1,2}(G)$ of the problem

$$
\begin{gathered}
\Delta v=0 \quad \text { on } G \\
\frac{\partial v}{\partial n}+v f=0 \quad \text { on } \partial G
\end{gathered}
$$

and $f \geq 0$, we obtain

$$
0=\int_{G} \nabla v \cdot \nabla v d \mathscr{H}_{m}+\int_{\partial G} v f v d \mathscr{H} \geq \int_{G}|\nabla v|^{2} d \mathscr{H}_{m} \geq 0 .
$$

Therefore, $\nabla v=0$ on $G$ and there is a constant $c$ such that $v(x)=c$ for $\mathscr{H}_{m}$-a.a. $x \in$ $G$ by [16, Corollary 2.1.9]. Since $\nu \in \mathscr{C}_{b}^{\prime}(\partial G)$, the function $U_{\nu}$ is bounded in $G$. Since $u(x)=\boldsymbol{U}\left(g \mathscr{H}_{m}\right)(x)+\mathcal{U} \nu(x)-c$ for $\mathscr{H}_{m}$-a.a. $x \in G$ and $\mathcal{U}\left(g \mathscr{H}_{m}\right) \in \mathscr{C}\left(\mathbb{R}^{m}\right)$ by Lemma 3, we obtain $u \in L_{\infty}(G)$.

\section{Acknowledgment}

The author was supported by GAČR Grant no. 201/00/1515.

\section{References}

[1] M. Brelot, Éléments de la Théorie Classique du Potentiel, Les Cours de Sorbonne. 3e cycle, Centre de Documentation Universitaire, Paris, 1959.

[2] B. E. J. Dahlberg, Estimates of harmonic measure, Arch. Rational Mech. Anal. 65 (1977), no. 3, 275-288.

[3] L. E. Fraenkel, An Introduction to Maximum Principles and Symmetry in Elliptic Problems, Cambridge Tracts in Mathematics, vol. 128, Cambridge University Press, Cambridge, 2000.

[4] L. L. Helms, Introduction to Potential Theory, Pure and Applied Mathematics, vol. 22, WileyInterscience, New York, 1969.

[5] H. Heuser, Funktionalanalysis, Mathematische Leitfäden, B. G. Teubner, Stuttgart, 1975.

[6] J. Král, Integral Operators in Potential Theory, Lecture Notes in Mathematics, vol. 823, SpringerVerlag, Berlin, 1980.

[7] N. S. Landkof, Fundamentals of Modern Potential Theory, Izdat. Nauka, Moscow, 1966.

[8] W. McLean, Strongly Elliptic Systems and Boundary Integral Equations, Cambridge University Press, Cambridge, 2000. 
[9] D. Medková, The third boundary value problem in potential theory for domains with a piecewise smooth boundary, Czechoslovak Math. J. 47(122) (1997), no. 4, 651-679.

[10] Solution of the Robin problem for the Laplace equation, Appl. Math. 43 (1998), no. 2, 133-155.

[11] I. Netuka, Generalized Robin problem in potential theory, Czechoslovak Math. J. 22(97) (1972), 312-324.

[12] An operator connected with the third boundary value problem in potential theory, Czechoslovak Math. J. 22(97) (1972), 462-489.

[13] - The third boundary value problem in potential theory, Czechoslovak Math. J. 22(97) (1972), 554-580.

[14] M. Schechter, Principles of Functional Analysis, Academic Press, New York, 1973.

[15] K. Yosida, Functional Analysis, Die Grundlehren der mathematischen Wissenschaften, vol. 123, Springer-Verlag, Berlin, 1965.

[16] W. P. Ziemer, Weakly Differentiable Functions, Graduate Texts in Mathematics, vol. 120, Springer-Verlag, New York, 1989.

Dagmar Medková: Mathematical Institute, Academy of Sciences of the Czech Republic, Žitná 25, 11567 Praha 1, Czech Republic

Current address: Department of Technical Mathematics, Faculty of Mechanical Engineering, Czech Technical University, Karlovo nám. 13, 12135 Praha 2, Czech Republic

E-mail address: medkova@math.cas.cz 


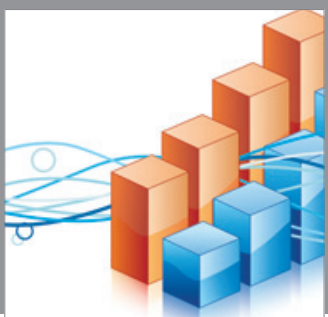

Advances in

Operations Research

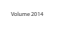

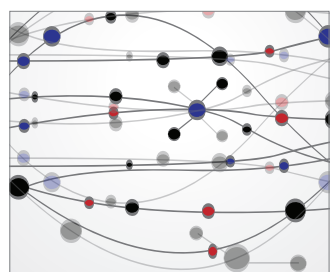

\section{The Scientific} World Journal
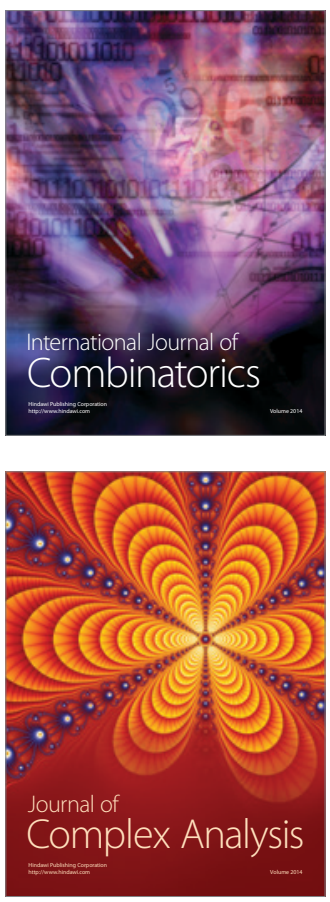

International Journal of

Mathematics and

Mathematical

Sciences
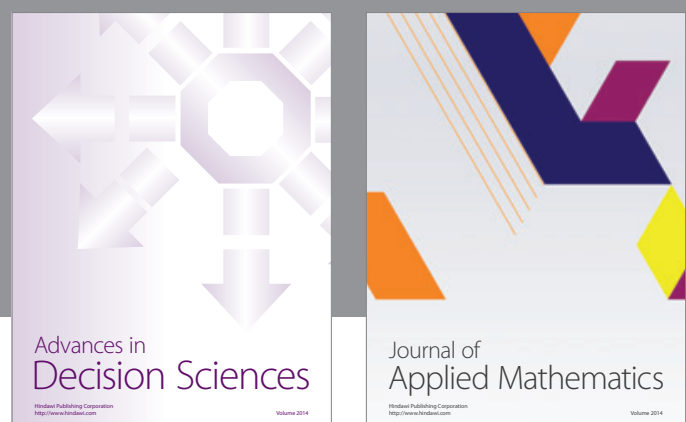

Journal of

Applied Mathematics
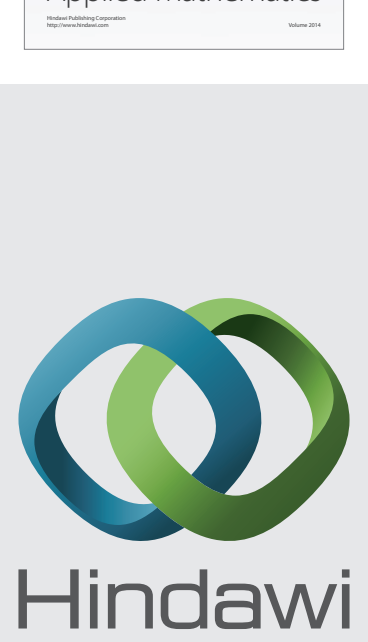

Submit your manuscripts at http://www.hindawi.com
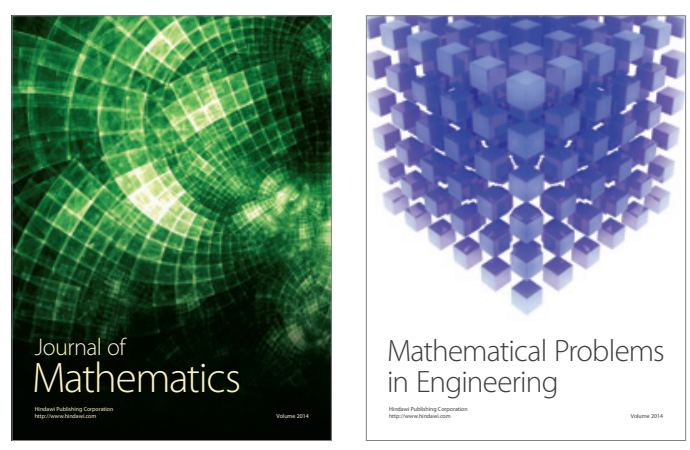

Mathematical Problems in Engineering
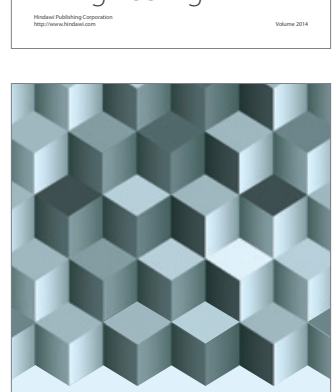

Journal of

Function Spaces
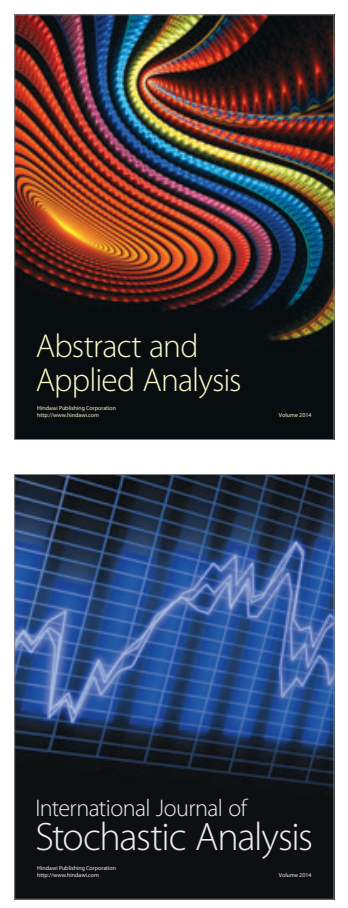

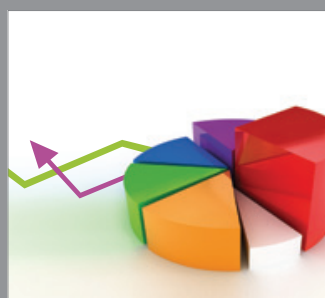

ournal of

Probability and Statistics

Promensencen
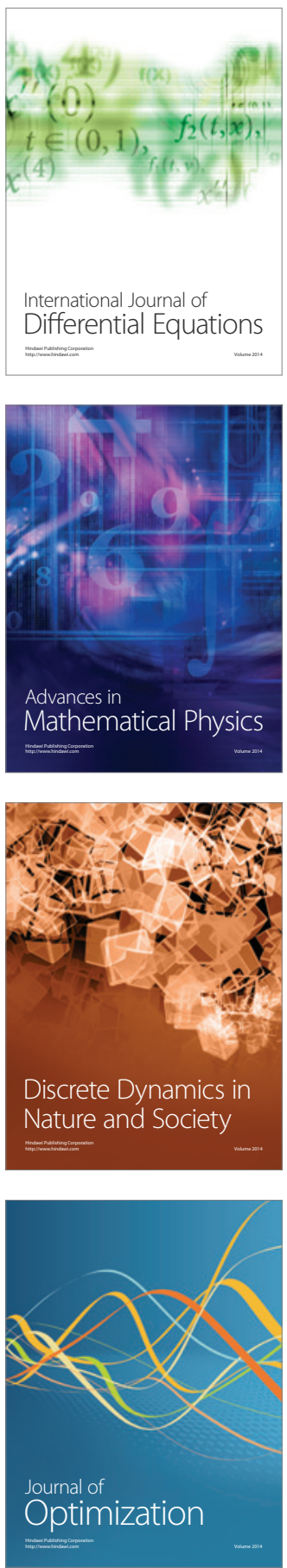